\title{
Gastric Cancer in Brunei Darussalam: Epidemiological Trend Over a 27 Year Period (1986-2012)
}

\author{
Vui Heng Chong ${ }^{1 *}$, Pemasari Upali Telisinghe ${ }^{2}$, Muhd Syafiq Abdullah ${ }^{1}$, Chee \\ Fui Chong ${ }^{3}$
}

\begin{abstract}
Background: Gastric cancer is the second most common gastrointestinal cancer and is more common in the East, compared to the West. This study assesses the trend of gastric cancers in Brunei Darussalam, a developing nation with a predominantly Malay population. Materials and Methods: The cancer registry from 1986 to 2012 maintained by the Department of Pathology, the only State Laboratory at the RIPAS Hospital, Ministry of Health, was reviewed and data extracted for analyses. The age standardised rate (ASR) and age specific incidence rate were calculated based on the projected population. Cancers diagnosed below 45 years were categorised as young gastric cancer. Results: Over the study period, there were a total of 551 cases of gastric cancer diagnosed. The most common type was adenocarcinoma $(87.9 \%)$, followed by lymphoma $(6.1 \%)$ and gastrointestinal stromal tumour (2.8\%). The overall mean age at diagnosis was 61.9 years old (range 15 to 98) with an increasing trend observed, but this was not significant (ANOVA). There were differences in the mean age at diagnosis for the different races $(p=0.003$ for trend), but not the gender $(p=0.105)$. Young gastric cancer accounted for $14.9 \%$, being more common in women, and in Expatriate and Malay populations compared to the Chinese. There was a decrease in the ASR, from 17.3/100,000 in 1986-1990 to 12.5/100,000 in 2006-2010. Chinese had a higher overall ASR (20.2/100,000) compared to the Malays (11.8/100,000). The age specific rates were comparable between men and women until the age group 55-59 years when the rates started to diverge, becoming higher in men. Chinese men had higher rates then Malay men whereas, the rates were higher or comparable between the women until the age group > 70 when the rate for Chinese women overtook their Malay counterpart. Conclusions: Our study showed that there is a declining trend in the incidence of gastric cancer and higher rates were observed in men and Chinese.
\end{abstract}

Keywords: Gastric neoplasm - trend - incidence - age adjusted rates - gastric cancers - Brunei Darussalam

Asian Pac J Cancer Prev, 15 (17), 7281-7285

\section{Introduction}

Gastric gastric cancer is the second most common cancer of the gastrointestinal tract, after colorectal cancer (IARC, Globocan 2012). In many countries, especially in developing countries, diagnoses are still made in the advanced stages. Symptoms of gastric cancer that include dyspepsia, nausea and discomfort are not-specific and resemble common gastrointestinal conditions such as peptic ulcer disease, non-ulcer dyspepsia and other upper gastrointestinal pathologies. When warning symptoms such as weight loss, dysphagia and vomiting manifest, the disease usually has been present for a considerable amount of time. Early diagnosis can be made through early investigations, but this is not possible in many centres due to the patient load or lack of screening programs. Screening programs such as those seen in Japan (Sano et al., 2014) and South Korea (Suh et al., 2013) have led to detection of cancer at the very early stages making curative treatment possible even with just endoscopic resection.

Risk factors for gastric cancer include Helicobacter pylori (H. pylori) infection, gastric mucosal atrophy, high intake of salted food and ageing (Bertuccio et al., 2013; Cover et al., 2013; de Martel et al., 2013). The pathogenesis of gastric cancer is well established and goes through a sequence of changes from chronic inflammation to atrophy, intestinal metaplasia, dysplasia and carcinomatous transformation (Bertuccio et al, 2013).

With the improvement in the standard of living and declining prevalence of $H$. pylori infection, the rates of gastric cancers have been reported to be decreasing (IARC, Gobocan 2012; Atrkar-Roushan et al., 2013). However, proximally located gastric cancers continue to increase and overall gastric cancer continues to be an important cause of cancer mortality (Deans et al., 2011). To date, there has been no published study looking at gastric cancer in Brunei Darussalam. This aims of this study were to a) assess the trend of gastric cancers over a 27 years period, 
and $b$ ) assess the differences between the major ethnic groups and genders.

\section{Materials and Methods}

Setting: Brunei Darussalam is a small developing nation with a total area of $5,765 \mathrm{~km}^{2}$ and estimated population of 415,717 (July 2013; Department of Economic Planning, Ministry of Finance) located on the island of Borneo. The population breakdown consists of ethnic group (Malays 66.3\%, ethnic Chinese 11\% and indigenous $2.7 \%$ ) and the expatriate group (20\%). The expatriate group consist mainly of those from Indian Subcontinent and the neighboring Southeast Asia countries; the Philippines, Indonesia, Thailand and Malaysia. The country is divided into four districts and each district has a government hospital supported by many outpatient clinics. Of the four districts, only two hospital (RIPAS Hospital and Suri Seri Begawan Hospital are equipped with endoscopic equipment). A private hospital also has provides endoscopy but only on a smaller scale. All tissue obtained for histology are sent to the State Pathology Laboratory located in the RIPAS Hospital (the only tertiary centre in the country with 550 beds).

The State laboratory maintains a cancer registry that started in 1984 until present. In this registry, only histology proven cancers are registered. Being the only state histopathology laboratory, the registry is for the whole country and therefore is representative. Data on age in the year 1984 and 1985 were not available, and these hence were excluded in the calculation of the Age Standardised Rate (ASR) and Age Specific Rate. The ASR and Age Specific Rate were calculated based on the projected population adjusted to the estimated world population breakdown. The annual ASR and five years block ASR (1986-1990, 1991-1995, 1996-2000, 2001-2005 and 20062010) were calculated. The ASR for 2011-2012 was also calculated. The overall ASR for the Malays and Chinese, the two major local groups were also calculated. The age specific rate (using five year grouping) for the genders was calculated based the national population breakdown in 1999.

The data were entered into the Wordexcel (Microsoft) and later transferred into the Statistical Package for Social Sciences (SPSS, Version 16.0, Chicago, IL USA) programme for analysis. We compared the differences between the genders, ethnic groups and also between young and old cancers. Chi square test was used to compare the categorical variables and the Student $t$ test and ANOVA were used to compare the continuous variables. A p value of less than 0.05 was taken as significant.

\section{Results}

Over the study period, there were a total of 551 cases of gastric cancer diagnosed. There were more men than women and more Chinese affected than the other racial group. The most common cancer was adenocarcinoma $(87.9 \%)$, followed by lymphoma $(6.1 \%)$ and gastrointestinal stromal tumour $(2.8 \%)$. The demographic and breakdown of the types of cancer is shown in Table 1.

The overall mean age at diagnosis was $61.9 \pm 15.6$ years old with an increasing trend observed, but this was not significant (ANOVA, $\mathrm{p}=\mathrm{ns}$ ). No significance differences in age at diagnosis were between the genders (Table 2). However, the expatriate group was younger at diagnosis (48.1 \pm 16.6$)$ compared to the other race ( $\mathrm{p}=0.003$ for trend)

Young cancer accounted for $14.9 \%$ of all the gastric cancers encountered during the 27 years period. Over the period, downward trend was observed in the proportion of young gastric cancer (Figure 2).

Among those categorized as young cancers, there were more women $(18.4 \%)$ compared to men $(13 \%)$, but this was statistically not significant $(\mathrm{p}=0.09)$. Young cancer was more common among the Expatriate group (57.1\%) compared to the Malays (15\%), Indigenous (14.3\%), and

Table 1. Demographic and Types of Cancers (1986 to 2012)

\begin{tabular}{|c|c|c|}
\hline \multicolumn{2}{|l|}{ Parameters } & $\mathrm{n}(\%)$ \\
\hline Age groups & $15-19$ & $1 \quad(0.2)$ \\
\hline & $20-24$ & $4 \quad(0.7)$ \\
\hline & $25-29$ & $12(2.2)$ \\
\hline & $30-34$ & $12(2.2)$ \\
\hline & $35-39$ & $25 \quad(4.4)$ \\
\hline & $40-44$ & $28 \quad(4.9)$ \\
\hline & $45-49$ & $38 \quad(6.6)$ \\
\hline & $50-54$ & $46 \quad(8.0)$ \\
\hline & $55-59$ & $56 \quad(9.8)$ \\
\hline & $60-64$ & $82(14.3)$ \\
\hline & $65-69$ & $56 \quad(9.8)$ \\
\hline & 70 and above & $190(33.2)$ \\
\hline \multirow[t]{2}{*}{ Gender } & Men & $368(64.3)$ \\
\hline & Women & $204(35.7)$ \\
\hline \multirow[t]{4}{*}{ Race } & Malays & 409 (71.5) \\
\hline & Chinese & $105(18.4)$ \\
\hline & Indigenous & $43 \quad(7.5)$ \\
\hline & Others/Expatriates & $15(2.6)$ \\
\hline \multirow[t]{6}{*}{ Tumour types } & Adenocarcinoma & $503(87.9)$ \\
\hline & Lymphoma & $35 \quad(6.1)$ \\
\hline & GIST/Leiomyosarcoma & $16(2.8) / 7(1.2)$ \\
\hline & Neuroendocrine & $5 \quad(0.9)$ \\
\hline & Squamous cell carcinoma & $5 \quad(0.9)$ \\
\hline & MFH & $1 \quad(0.2)$ \\
\hline
\end{tabular}

GIST: Gastrointestinal stromal tumor; MFH: Malignant fibrohistocytoma

Table 2. Mean Age at Diagnosis of Gastric Cancers between the Genders, Racial Groups and Tumor Types

\begin{tabular}{llcc}
\hline Variables & & \multicolumn{2}{c}{$\begin{array}{c}\text { Mean age (years) } \\
\text { and standard deviation }\end{array}$} \\
\hline Genders & Men & $62.68 \pm 15.15$ & 0.105 \\
& Women & $60.43 \pm 16.19$ & \\
Racial groups & Malays & $61.64 \pm 15.74$ & 0.003 \\
& Chinese & $64.60 \pm 13.69$ & \\
& Indigenous & $62.10 \pm 15.57$ & \\
Tumor types* & Expatriates & $48.14 \pm 16.81$ & \\
& Adenocarcinoma & $62.71 \pm 15.31$ & 0.003 \\
& Lymphoma & $54.71 \pm 15.49$ & \\
& GIST & $61.56 \pm 16.88$ & \\
& Leiomyosarcoma & $48.29 \pm 16.58$ & \\
& Neuroendocrine & $62.40 \pm 17.01$ & \\
& Squamous cell & $46.75 \pm 9.60$ & \\
\hline
\end{tabular}

* Less common types of gastric cancers not included 
lowest among the Chinese (8.9\%) with a significant trend $(\mathrm{p}<0.001)$.

The annual ASR showed a fluctuated downward trend from $18.90 / 100,000$ in 1986 , peaking in 1987 $(22.7 / 100,000)$ to $7.78 / 100,000$ in 2012 (Figure 3a). The overall ASR in five years block is shown in Figure $3 \mathrm{~b}$.

Chinese had higher ASR $(20.18 / 100,000)$ compared to the Malays $(11.83 / 100,000)$.

The age specific incidence rates were higher among men compared to women (Figure 4). The rates were similar until the age group 45-49 when the rates started to diverge. However for the age group 45-49, the rate was slightly higher in women before, the rate became marked different, progressively higher in men.

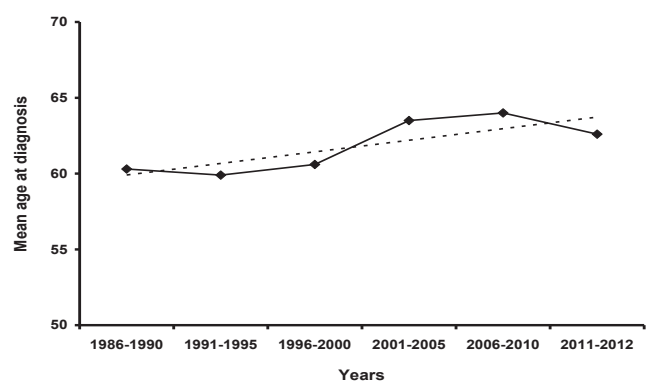

Figure 1. The Mean Age at Diagnosis of Gastric Cancer Over the Study Period (1986 to 2012)

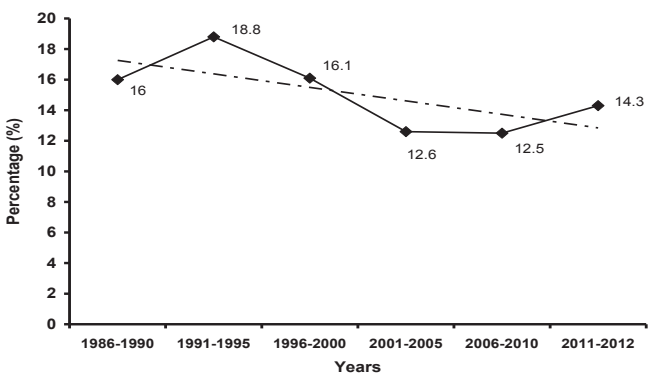

Figure 2. Proportion of Young Gastric Cancer (defined as Younger then 45 years) from 1986 to 2012

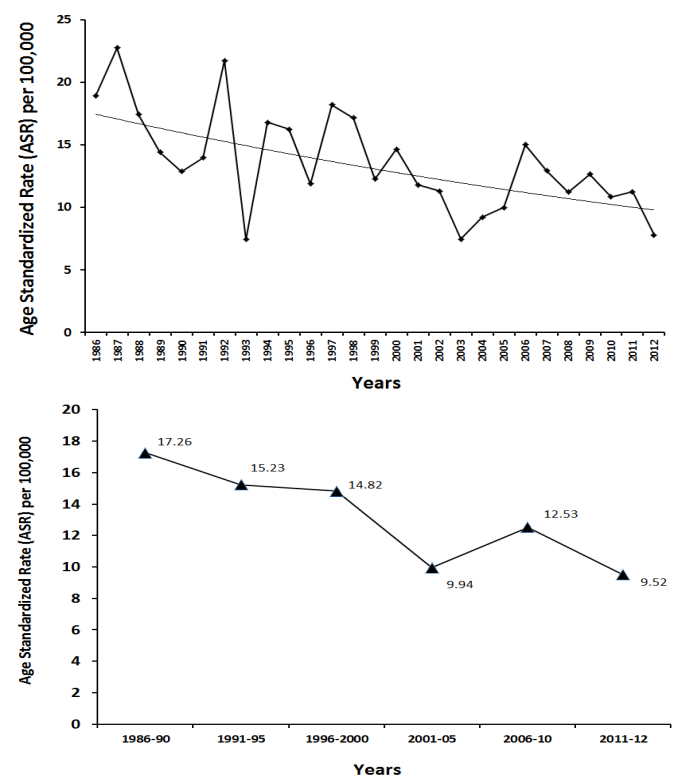

Figure 3. The ASR for Gastric Cancer for Individual Years (1986 to 2010) and the Five Figures is for Year 2011-2012
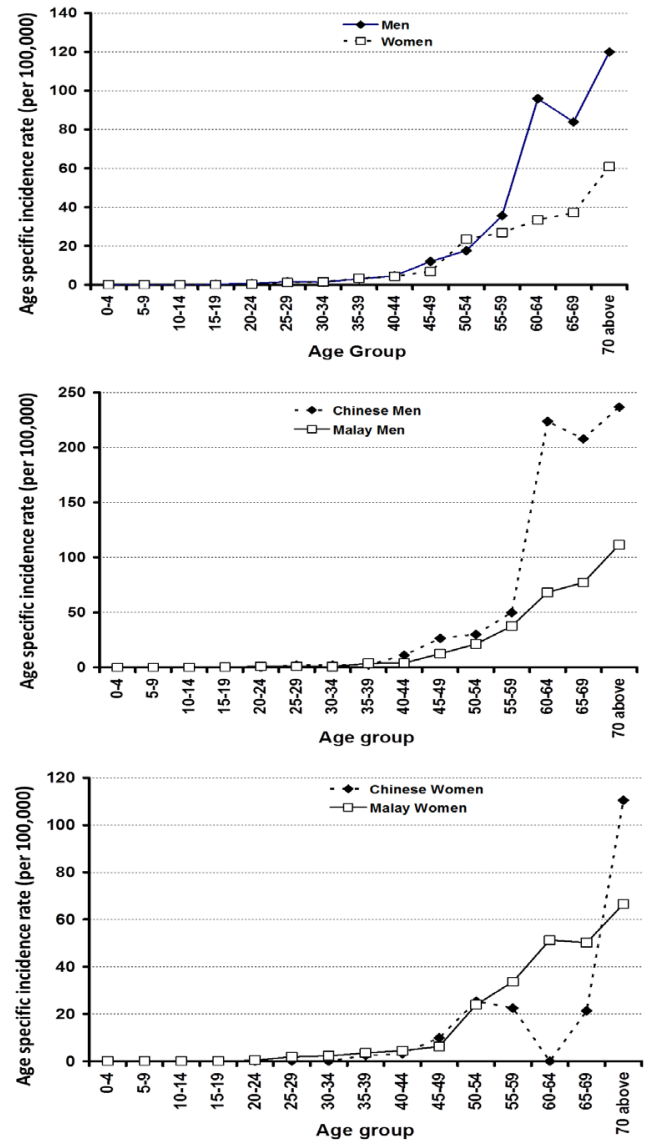

Figure 4. The Age Specific Incidence rates for Gastric Cancers between the Genders, Malay and Chinese Men and Women respectively

Between the two major ethnic groups, Chinese men had rates higher than their Malay counterpart and the curve started to diverge from the 40-44 years age group but became more pronounced after the 55-59 years group (Figure 5). As for the women, the Malay women had higher rates until the age group 65-69 years (Figure 6). There were no cases recorded for Chinese women in the age group 60-64 years.

\section{Discussion}

Overall the study period, there were 551 cases of histologically proven gastric cancers encountered. While our number is small compared to the numbers reported by other countries (IARC, Gobocan 2012), our population is small. The histological types of gastric cancer diagnosed in Brunei Darussalam are similar to what have been reported with the most common tumor type being adenocarcinoma, followed by lymphoma and gastrointestinal stromal tumor.

Over the 27 years period, there was slow increase in the mean age, reflecting the ageing population. Importantly, there was a steady decline in the ASR, consistent to what have been reported elsewhere (Look et al., 2001; IARC, Globocan 2012; Atrkar-Roushan et al., 2013). H. pylori is a very important factor in the pathogenesis of gastric cancer and certain bacterial virulent characteristics virulent characteristics are very important (Cover et al., 2013; Sepulveda., 2013). Ever since its discovery 
H. pylori in the early 1980 s, our understanding of the pathogenesis of gastric cancers has improved (Pandey et al., 2010). Widespread practice of test and treat have led to declining prevalence of this infection (Lim et al., 2013; van Blankenstein et al., 2013), including Brunei Darussalam (Chong et al., 2008) have also led to the decline in the incidence of gastric cancer. Even though the prevalence of H.pylori has decline, approximately one sixth to a fifth of our patients still have this infections (Chong et al., 2008).

There were also differences in the incidence rates between the genders and racial groups. Men generally had higher incidence of gastric cancers and this is seen in most countries (IARC, Globocan 2012). A possible explanation for this is the higher exposure to risk factors, including higher prevalence of $H$. pylori infection (Chong et al., 2008; Lim et al., 2013; van Blankenstein et al., 2013), smoking (Goh et al., 2007), alcohol consumption and dietary habits (high intake of salted fish and vegetable increase the risk while high fruits and vegetable intake being protective) (Goh et al., 2007). Many other environmental factors have been implicated. Between the two major racial groups, Chinese had ASR almost twice compared to the Malays. The difference was more apparent among the male gender. Our findings are consistent to what have been reported in Malaysia (Goh, 2009) and Singapore (Fock et al., 2010), two Southeast Asia nations that similar ethnic groups. In these two countries, the Chinese race has higher prevalence of gastric cancers and $H$. pylori infection. Low incidence among Indian despite prevalence of $H$. pylori infection suggest other factors (Goh et al., 2007), one being the genetic make-up (Figueiredo et al., 2013).

This was higher among men, and between the two major racial groups, Chinese men had higher rates after the age of 40 . This difference was not seen among the women. The rates were comparable between the Chinese and Malay women, but higher in Malays in the 54 to 69 age group. Chinese women had higher rate after 70 . The fluctuation we observed between the Chinese and Malay women is likely due to the relative small number of cases.

Like many cancers, even the younger population are affected. In our cases, young gastric cancers accounted for $14.9 \%$ of all the gastric cancers. More women were affected compared to men. Young cancers in general tend to be more aggressive especially for the adenocarcinoma types. However, in our case young cancers was mainly due to lymphoma which has quite favorable treatment outcomes. Even so, the proportion of young gastric cancer is still high. Among the racial groups, the Expatriate group had the highest rate of young gastric cancers. Prior to starting their employment, they go through mandatory medical fitness testing and given the population pyramid population, almost all within the working age; it is not surprising that more than half of the gastric cancers diagnosed were considered young cancer. Overall, the absolute number of gastric cancer in this group was small.

Even though the incidence of gastric cancer is declining, it is still an important cause of cancer related morbidity and mortality. Importantly, most of our gastric cancers are still diagnosed in advanced or locally advanced stages. Therefore, more needs to be done to overcome this. Given that $H$. pylori is a Class I carcinogen, we will continue to practice the test and treat strategy for all patient undergoing endoscopy. Other interventions that need to be considered include increasing public awareness on the risk factors (H. pylori, smoking, alcohol and diet), improvement in lifestyle and presenting early. Screening for gastric cancers has been shown to be effective in Japan (Sano et al., 2014), South Korea (Suh et al., 2013) and China (Lu et al., 2014), countries where the incidence of gastric cancer is high. However, in our setting, this may be not cost-effective given the lower incidence. Selective screening such following up patient with documented gastric intestinal atrophy and metaplasia may be more cost effective. Use of non-endoscopic screening as such using serum markers (gastrin and pepsinogen I/II ratio) (Shafaghi et al., 2013) or other novel markers can be further studied and considered (He et al., 2013).

In conclusion, our study showed that there is a declining trend in gastric cancer over a period of almost three decades. The rates were higher rates in men and the Chinese. Our findings are consistent to those reports from other countries.

\section{References}

Atrkar-Roushan Z, Kazemnejad A, Mansour-Ghanaei F, Zayeri F (2013). Trend analysis of gastrointestinal cancer incidences in Guilan province: comparing rates over 15 years. Asian Pac J Cancer Prev, 14, 7587-93.

Bertuccio P Grabsch HI, Tan P (2013). Gastric cancer pathology and underlying molecular mechanisms. Dig Surg, 30, 150-8.

Bertuccio P, Rosato V, Andreano A, et al (2013). Dietary patterns and gastric cancer risk: a systematic review and metaanalysis. Ann Oncol, 24, 1450-8.

Chong VH, Lim KC, Rajendran N (2008). Prevalence of active Helicobacter pylori infection among patients referred for endoscopy in Brunei Darussalam. Singapore Med J, 49, 42-6.

Cover TL, Peek RM Jr (2013). Diet, microbial virulence, and Helicobacter pylori-induced gastric cancer. Gut Microbes, 4, 482-93.

de Martel C, Forman D, Plummer M (2013). Gastric cancer: epidemiology and risk factors. Gastroenterol Clin North Am, 42, 219-40.

Deans C, Yeo MS, Soe MY, et al (2011). Cancer of the gastric cardia is rising in incidence in an Asian population and is associated with adverse outcome. World J Surg, 35, 617-24.

Fock KM, Ang TL (2010). Epidemiology of Helicobacter pylori infection and gastric cancer in Asia. J Gastroenterol Hepatol, 25, 479-86.

Goh KL (2009). Epidemiology of Helicobacter pylori infection in Malaysia--observations in a multiracial Asian population. Med J Malaysia, 64, 187-92.

Goh KL, Cheah PL, Md N, Quek KF, Parasakthi N (2007). Ethnicity and $H$. pylori as risk factors for gastric cancer in Malaysia: A prospective case control study. Am J Gastroenterol, 102, 40-5.

He CZ, Zhang KH (2013). Serum protein and genetic tumor markers of gastric carcinoma. Asian Pac J Cancer Prev, 14, 3437-42.

International Agency for Research on Cancer (IARC). GLOBOCAN 2012. www.globocan.iarc.fr/ (accessed 15th April 2014).

Lim SH, Kwon JW, Kim N, et al (2013). Prevalence and risk factors of Helicobacter pylori infection in Korea: nationwide multicenter study over 13 years. BMC Gastroenterol, 13, 
104.

Look M, Gao F, Low CH, Nambiar R (2001). Gastric cancer in Singapore. Gastric Cancer, 4, 219-22.

Lu YF, Liu ZC, Li ZH, et al (2014). Esophageal/gastric cancer screening in high-risk populations in Henan Province, China. Asian Pac J Cancer Prev, 15, 1419-22.

Figueiredo C, Garcia-Gonzalez MA, Machado JC (2013). Molecular pathogenesis of gastric cancer. Helicobacter, 18, 28-33.

Pandey R, Misra V, Misra SP, et al (2010). Helicobacter pylori and gastric cancer. Asian Pac J Cancer Prev, 11, 583-8.

Shafaghi A, Mansour-Ghanaei F, Joukar F, et al (2013). Serum gastrin and the pepsinogen I/II ratio as markers for diagnosis of premalignant gastric lesions. Asian Pac J Cancer Prev, 14, 3931-6.

Sano H, Goto R, Hamashima C (2014). What is the most effective strategy for improving the cancer screening rate in Japan? Asian Pac J Cancer Prev, 15, 2607-12.

Sepulveda AR (2013). Helicobacter, inflammation, and gastric cancer. Curr Pathobiol Rep, 1, 9-18.

Suh M, Choi KS, Lee YY, Park B, Jun JK (2013). Cancer screening in Korea, 2012: results from the Korean National Cancer Screening Survey. Asian Pac J Cancer Prev, 14, 6459-63. 